\title{
Subject Index Vol. 69, 1996
}

B-Adrenoceptor 383 Albuterol 140 Antioxidants 284 o $1 / 8$-Antiplasmin 298 Arachidonic acid metabolites 101 Astrocytes 327 Atherogenesis 109 Atrial natriuretic peptide 60 Autonomic nervous system 22

Baclofen, anticonvulsant action

405 Bilirubin encephalopathy 268

kinetics 268 Blood flow 101

T lymphocytes 128

Cardiorespiratory system 146 ß-Carotene 230 Catecholamines 383 CD34 1

CD45isoforms 128 Cerebral blood flow 284

circulation 357 cGMP 60 Chloral hydrate 146 Chromogranin A 22 Cold 51 Complement 225 receptors 225 Compliance 275 Congenital diaphragmatic hernia

94,318 Cortical field 399 Cytodifferentiation 399 Cytoskeleton 257

Decay-accelerating factor 225

Degranulation 368

5'-Deiodinase 51

Delivery 30

Dendrites 257

Development, hypoxia-ischemia

327 Diabetes 37,293 Diaphragm 67

DNA 153

Dopamine B-hydroxylase 22

Doppler echocardiography 243

Eicosanoids 153 Electrocortical activity 284 Electrolyte transport 60 Electrophoresis 67

Embryopathy 293 Endothelium-derived relaxing

factors 389 Energy intake 51 Epigenetic cues 399 Experimental respiratory distress

syndrome 275 Eye 101

Fc $\gamma$ RIII 235 Femoral artery 153 Fibrinolysis 298 Flow cytometry 235 Full-term newborns 76

Functional murmur 243

Gas exchange 275

Gene expression 342

Genioglossus 67

Glomerular microcirculation 389

Glucagon 307

Glucocorticoids 342

Glutathione 293

Granulocytes 368

Growth hormone 76, 307

- - releasing factor 307 
Haemoglobin 350 Heart rate 350 Hemolytic anemia 268 Hippocampus 257 Human colostrum 376 Hyperkalemia 140 Hyperoxia 101 Hypoxia 146 -, piglet 350

Hypoxia-ischemia, rat model 327

Hypoxic-ischemic encephalopathy 76

Ibuprofen 101 Immature kidney 389 Instillation method 119 Insulin 307 Interleukins 1, 376

Intracranial bleeding 357 Intrauterine growth retardation 307

Kernicterus 268

Lactate 350

Liquid ventilation 275

Lung immaturity 94,318

Maternal-fetal transfer 230 Membrane cofactor protein 225 Mesenteric artery 153 Methionine enkephalin 22 Microflora 30 Microglia 327 Mononuclear phagocytes 1 Motor cortex 399

Myocardium, rat 383 Myosin heavy chain 67

$\mathrm{Na}+\mathrm{K}+\mathrm{ATPase} 140$ Neonatal blood 1 Newborn infants, CD45 isoforms 128

- piglet 146

NG-nitro-L-arginine methyl ester

84 Nitric oxide 389

$-$

Nitrofen 94

- inhalation 284

Northern Canadians 133

Ontogenesis, rat 383 Organogenesis 12 Oxidative stress 293 Oxygen 101

415

Pentylenetetrazol 405 Perfluorocarbons 275 Perinatal asphyxia 76

- $\quad$ stress 109

Peripheral blood mononuclear

cells 376 Periventricular leukomalacia 327 Peroxisomal enzymes 12 Peroxisomes 12

Physiological stenosis, pulmonary

artery 243 Piglet coronaries 109 Placenta 230 Placental leucine aminopeptidase

307 Plasma retinol 133 Plasminogen 298 Platelet-activating factor 119 Polymorphonuclear

neutrophils

235 Polyunsaturated fatty acids 368 Postnatal development, rabbit 60

- $\quad$ intestinal development, mice

342

Pregnancy 37, 293 Premature infants 235

lambs 275

newborns 76 Prematurity 327

Prenatal dexamethasone treatment 318

- $\quad$ hypoxia 383

Preterm infants, $\mathrm{O} 2$ tension 357

Progenitor cells 1

Prolactin 76

Protein 153

Proteolysis 257

Pulmonary distribution 119

hypoplasia 94,318

vascular resistance 84 
Rat development, baclofen 405

liver 37 Respiratory burst 368

distress syndrome 119 Retina 101

Retinol 230

Retinopathy, prematurity 101 Rubidium 140

Seizures, baclofen 405 Sepsis 249 Small intestine 12 Somatostatin 307 Staphylococcus

epidermidis colonization 249 Steroids 249 Stroke 257 Surfactant 94, 275

substitution 119

therapy 318

Systemic vascular resistance 84

Thyroid hormone receptors 51 Transcription factors, fos, jun 342 Translation, initiation factors 37

Vagina 30 Vasomotor tone 84 Vitamin A intake 133

- $\quad$ - status 230

416

Subject Index Vol. 69, 1996 